\title{
Transport in Porous Media with Nonlinear Flow Condition
}

\author{
Mohaddeseh Mousavi Nezhad $^{1}$ - Mohammad Rezania ${ }^{1}$ • Elisa Baioni ${ }^{1}$
}

Received: 24 June 2018 / Accepted: 9 October 2018 / Published online: 25 October 2018

(c) The Author(s) 2018

\begin{abstract}
We investigate local aspects and heterogeneities of porous medium morphology and relate them to the relevant mechanisms of momentum transfer. In the inertial flow range, there are very few experimental data that allow to recognize the effects of porous structure on the flow and transport through porous media. An experimental analysis was performed in order to understand above processes at different Reynolds numbers in randomly structured porous media. The objective of the analysis is to explore the effects of porous media particle size on inertial and viscous forces and determine range of the Reynolds numbers in which the inertial flow predominantly contributes in dispersive processes. Transport characteristics of the randomly structured porous media and the influence of inertial force on longitudinal and transverse dispersion coefficients were studied.
\end{abstract}

Keywords Inertial flow · Stochastic finite element · Uncertainty quantification · Heterogeneous porous media and nonlinear flow

\section{Introduction}

A hydrodynamic dispersion coefficient is the sum of a diffusion coefficient and a mechanical mixing coefficient. Slichter (1905) was the first to show interest in dispersion investigations and concluded that velocity variation is the principle cause of dispersion. The dispersion process shows a complex behaviour in porous media with uncertain flow field as it depends on the microscopic pattern of flow through porous media, and a correlation between Reynolds number and the dispersion is expected to exist.

In primary studies on dispersion (e.g. Day 1956; Scheidegger 1954), it was assumed that a perfect mixing in fluid flow through a system occurs with a constant rate, which is not always true in real natural or engineering porous media with heterogeneous microstructure. Velocity variations and pressure excitations caused by heterogeneity of porous media make the transport process within this kind of non-uniform structure anomalous and more complicated. Scheidegger (1961) proposed a general theory, which mathematically describes the dispersion mechanism of a viscous fluid moving through a porous media with random geometrical properties. Brenner (1980) used the approaches introduced by Aris (1956) to model

Mohaddeseh Mousavi Nezhad

m.mousavi-nezhad@warwick.ac.uk

1 School of Engineering, The University of Warwick, Coventry CV4 7AL, UK 
the kinematics of flow in spatially periodic porous media based on Taylor (1953) on dispersion in cylindrical capillaries. Brenner (1990) also reviewed some subsequent developments on this Taylor-Aris model for macroscale transport processes, which is called the Taylor dispersion phenomena. This study illuminated elementary examples in macroscale modelling and physical interpretation of microscale convective-diffusive-reactive transport processes. Effects of some factors such as fluid viscosity, density, particle size distribution, shape of particles have also been investigated using packed column tests and experimental techniques of dispersion coefficients measurement (De Josselin de Jong 1958; Han et al. 1985; Delgado 2006; Çarpinlioğlu et al. 2009; Cheng 2011; Erdim et al. 2015; Allen et al. 2013; Majdalani et al. 2015; Vollmari et al. 2015; Hofmann et al. 2016; Norouzi et al. 2018).

Several theoretical and numerical techniques have been considered in order to incorporate the effects of these flow variations in the transport process modelling. For example: continuous time random walks (Montroll and Weiss 1965), integral transform methods (Cotta 1993), Taylor-Aris-Brenner moment methods (Shapiro and Brenner 1986, 1988), stochastic convective (Simmons 1982; Nezhad et al. 2011), central limit theorems (Evans and Kenney 1966) and homogenization methods (Sanchez-Palencia 1980). Most of these researches focus on understanding the dispersion process in heterogeneous porous media when the flow is within the Darcy regime (Nezhad and Javadi 2011; Nezhad et al. 2013; Dentz et al. 2004).

Fluid flow through porous media is governed by various forces that act on the fluid, including viscous forces, gravitational force, electromagnetic forces and forces due to pressure from surrounding fluid. Sum total of these forces is referred to as inertia force and according to the results of early researches on this field (i.e. Fand et al. 1987; Kececioglu and Jiang 1994; Bagci et al. 2014), depending on the amount of contribution of inertial and viscous forces in deriving flow, four different flow regimes are generated which are distinguished by the type of relationship between fluid velocity and pressure drop though porous media. These three regimes which are defined are namely pre-Darcy, linear Darcy, nonlinear Forchheimer and turbulent flow regimes.

Inertial forces can influence the hydraulic conductivity and dispersion (Van der Merwe and Gauvin 1971). LeClair and Hamielec (1968) investigated the viscous flow at intermediate Reynolds numbers. This work employed the finite difference methods from Hamielec et al. (1967a, b) and used the results to develop the theoretical information of local velocity distributions about particles for the intermediate flow regime and discussed its application to the packed beds. Forchheimer (Forchheimer 1901 cited in Whitaker 1996) was the first to consider the nonlinear relationship between hydraulic gradient and flux at larger velocities, and Prausnitz and Wilhelm (1957) and Mickley et al. (1965) presented theoretical and experimental relationship between turbulence parameters and concentration fluctuations in packed beds. However, there are only few research investigating this correlation. Bijeljic and Blunt (2006) and Haserta et al. (2011) have investigated the contribution of inertial flow on longitudinal dispersion. It was indicated that in realistic heterogeneous porous media, the dispersion coefficient is unlikely to reach an asymptotic value and traditional advection-dispersion equation cannot model solute transport (Nezhad 2010).

Despite numerous experimental and theoretical studies on dispersion there is a lack of fundamental understanding of how complexity in flow field in high Reynolds number can influence on the dispersion and how these processes are influenced by heterogeneity of the porous media. Boundary of flow regimes would be varied among different experimental systems, because of the geometry-dependent manifestation of inertial effects, particularly from the transition to turbulence (Reddy et al. 1998). Importance of specifying a transition boundary between these regimes where fluid field gradually evolves from linear to nonlinear, 
and from laminar to turbulent flow are recognized when appropriate method should be used for transport modelling and calculation of dispersion coefficient.

In this paper, the effects of nonlinearity of fluid flow on dispersion are investigated using a set of tracer tests conducted on packed beds of spherical glass beads of different diameter size. The effects of fluid characteristics on the flow behaviour and transition boundary between flow regimes have not been investigated in this work and will be done in future work.

\section{Experimental Setup}

The experimental system used in this project was originally designed at the Aachen University of Technology in Germany, and Chandler (2012) developed it to analyse the vertical variation in diffusion coefficient within sediments. This system has been modified for use in this research. It consisted of an upstream and a downstream circular column, with a sample column in between having a diameter of $96 \mathrm{~mm}$ and a length of $200 \mathrm{~mm}$. Figure 1 shows a schematic view of the modified system.

Soda lime glass spheres with diameter of $1.85 \mathrm{~mm}$ and $3 \mathrm{~mm}$ were used to make the sample columns. Both samples were compacted to have the same porosity of 0.39 . The spheres are non-reactive with Rhodamine WT which was used as dye for tracer experiments. The sample columns were placed on a stainless steel mesh with a pore size smaller than the particle diameters, in order to prevent spheres from clogging the outlets, avoiding any additional delay or fiction of the fluid flow. All the tests were done under the same temperature conditions and a temperature sensor and a Rapid 318 digital multimeter were attached to the downstream column wall to monitor the temperature in the system throughout experiments. The head difference was measured at two manometer tubes which were connected to upper and lower positions of the sample column. A WATSON MARLOW 505Di peristaltic pump was used to apply a steady flow to the system with constant operation temperature. The pump has the capacity to produce discharge rates of up to $18.95 \mathrm{~m}^{3} / \mathrm{s}$, producing water flow from Darcy to non-Darcy regimes. Air bubbles were removed before doing any tests, to guarantee vacuum conditions. Only water was used as fluid in this study and effect of fluid characteristics on the flow behaviour are left to be investigated in future work.

A joint was inserted between the inflow pipes and was used to inject Rhodamine water tracing dye (Rhodamine WT) into the system with a syringe. Two Cyclops 7 Submersible Fluorometers were attached to both upstream (inlet) and downstream (outlet) columns to continuously record the cross-sectional temporal dye concentration distribution during the tests. The data measured by the fluorometers were recorded automatically and stored electronically in a Novus LogBox-AA. All sensors were calibrated prior to use and details of the calibration procedure are found in Liang (2017).

\section{Results and Discussion}

\subsection{Flow Regime}

In order to investigate the effects of nonlinearity of fluid flow on the transport behaviour in porous media, the experiments were conducted under both linear and nonlinear flow regimes. Nonlinear plots of the experimental data describing pressure drop versus Reynolds number which are presented in Fig. 2 prove the existence of nonlinear flow. Reynolds number as a 


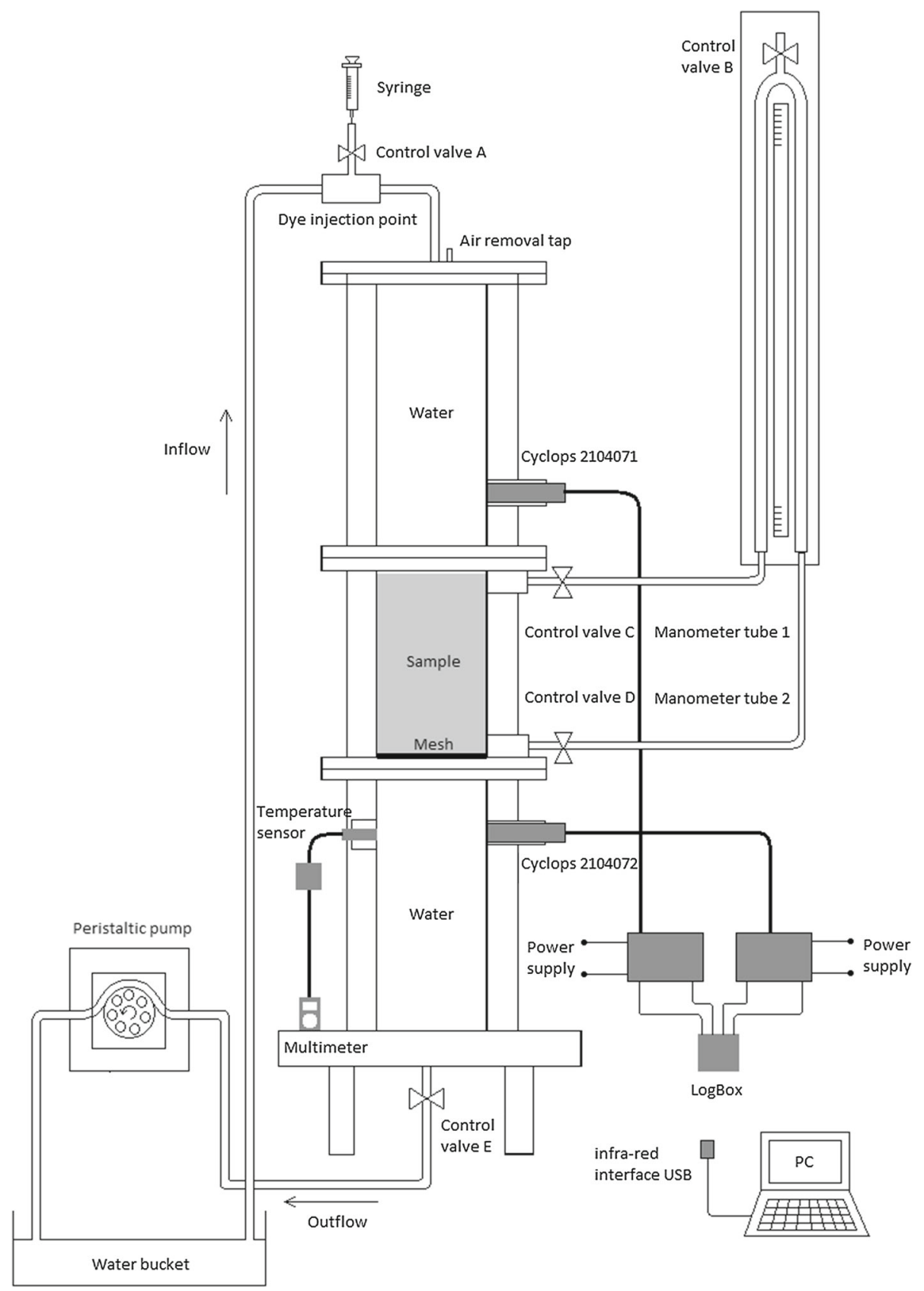

Fig. 1 Schematic of experimental system setup

factor to define the flow boundaries, it represents a ratio of inertial forces to viscous forces, indicates the change of flow regime when the inertial effect becomes important. Approaches for calculating the Reynolds number or for defining a criterion for specifying flow regimes 


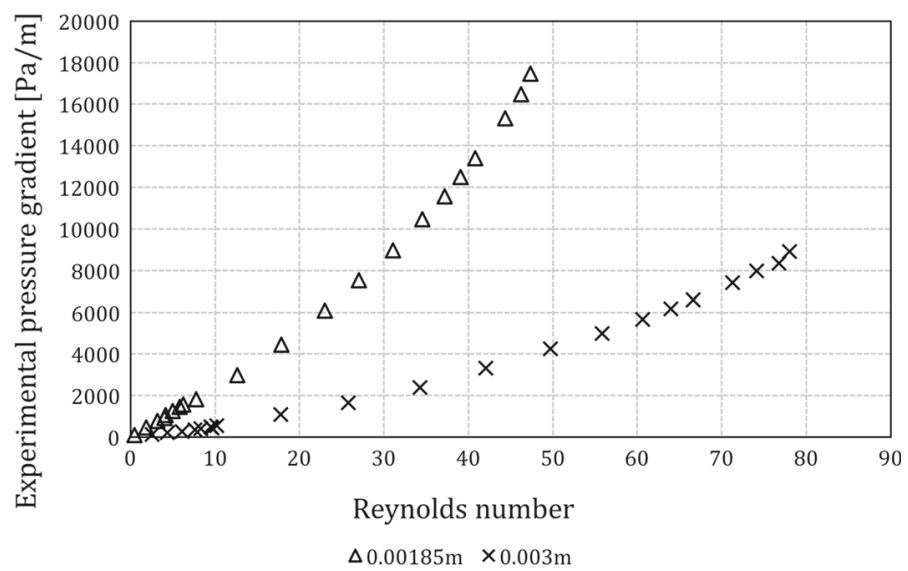

Fig. 2 Experimental pressure gradient versus Reynolds number for both $1.85 \times 10^{-3} \mathrm{~m}$ and $3 \times 10^{-3} \mathrm{~m}$ samples

in porous media are divergent and inconsistent, different authors had their own statements on distinguishing the critical zone of Darcy and non-Darcy flow, based on the comparison of experimental results and other literature (Zeng and Grigg 2006). In this work, Reynolds number were calculated as

$$
R_{\mathrm{eL}}=\frac{\rho v L}{\mu}
$$

where $\rho$ is water density $\left(\mathrm{kg} / \mathrm{m}^{3}\right), v$ is Darcy velocity $(\mathrm{m} / \mathrm{s}), \mu$ is dynamic viscosity $[\mathrm{kg} /(\mathrm{m} \mathrm{s})]$ and $L$ is characteristic length (m). The $L$ is defined as the inverse of specific interfacial area, the contact area of water and solid phase in unit volume (Petrasch et al. 2008).

It is seen that under the same flow conditions a higher-pressure drop is observed for the sample with smaller particles. Although these two samples have the same porosity, the passages of flow in the $1.85 \times 10^{-3} \mathrm{~m}$ sample are narrower and much more circuitous. Therefore, the pressure gradient across the sample with the smaller particles is more sensitive to the change of the flow condition.

Identification of the boundaries between different flow regimes is a challenge. Some criteria have been proposed in the literature to specify such boundaries. Following the methodology proposed by Kececioglu and Jiang (1994), plotting the dimensionless pressure gradient versus Darcian Reynolds number and identifying the tracts where the slope of lines changes, it is possible to identify different flow regimes as it is shown in Fig. 3. A linear regression was used to find the trend lines for the dimensionless plots. The points are selected in order to have a high value of $R^{2}$ (coefficient of determination) so that the regression line gives a good representation of the distribution of the points on the plot. It is shown that the Reynolds number $\left(R_{\mathrm{eL}}\right)$ corresponding to the transition from linear to nonlinear flow is 2.48 for $1.85 \times 10^{-3}$ $\mathrm{m}$ sample and 3.24 for $3 \times 10^{-3} \mathrm{~m}$ sample.

\subsection{Determination of Intrinsic Permeability and Forchheimer Coefficient}

Forchheimer equation with Ergun expression for Forchheimer coefficient is presented as

$$
-\frac{\Delta p}{L}=\frac{\mu}{k} v+\frac{\rho C_{\mathrm{E}}}{\sqrt{k}} v^{2}
$$




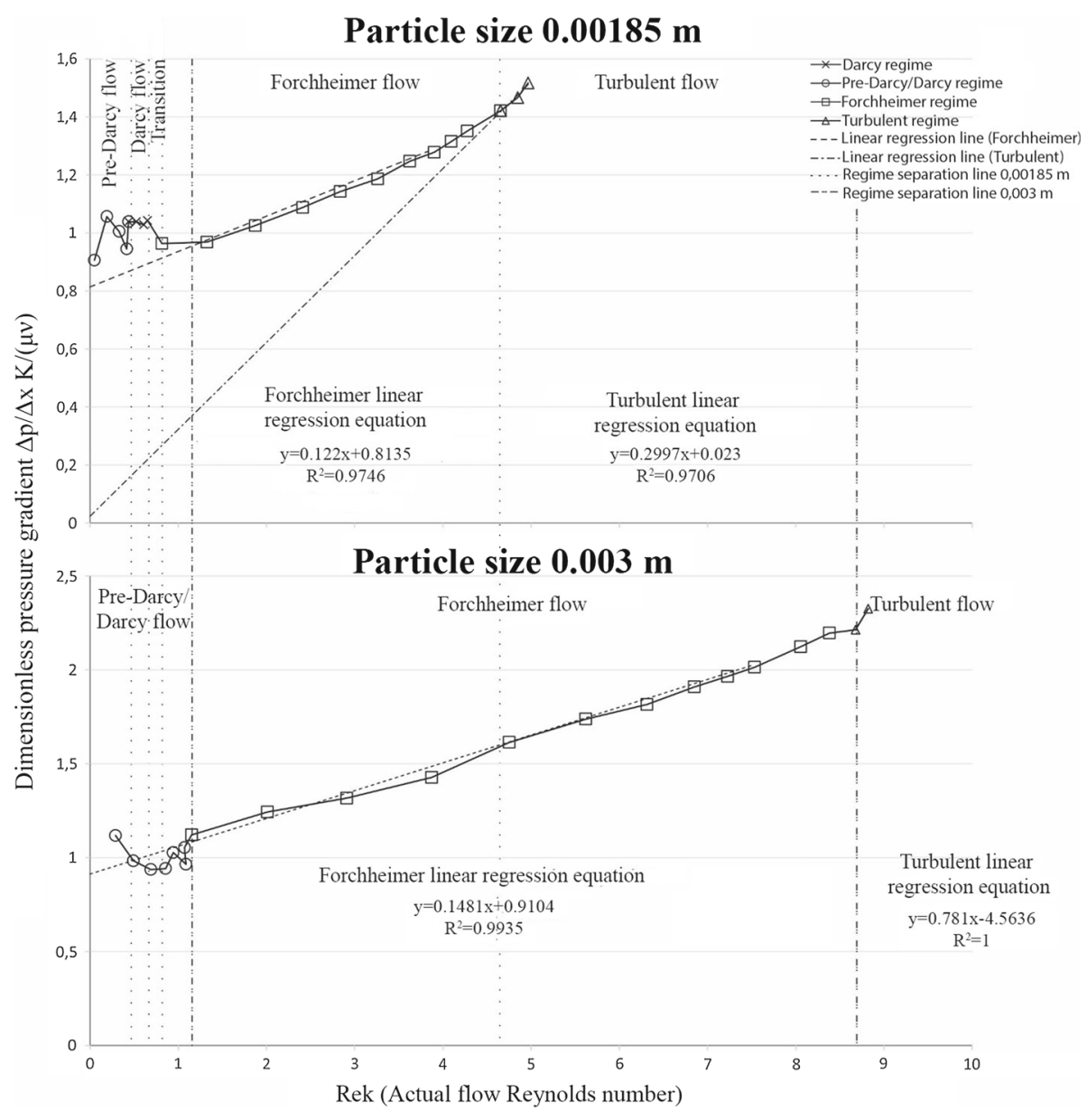

Fig. 3 Dimensionless pressure gradient and actual flow Reynolds number for $1.85 \times 10^{-3} \mathrm{~m}$ and $3 \times 10^{-3} \mathrm{~m}$ samples, distinction of different flow regime

where $C_{\mathrm{E}}$ is Ergun coefficient, which depends on the geometry of pore space distribution. Forchheimer coefficient $\beta$ is defined as a function of the Ergun coefficient, where $\beta=\frac{C_{\mathrm{E}}}{\sqrt{k}}$.

Forchheimer and Ergun coefficients in the Forchheimer equation were calculated initially by implementing a nonlinear regression of the experimental data. The calculation was performed in two ways: (a) using whole sets of experimental data (labelled as B1, B2, B3 in Fig. 4); and (b) using only those within Reynolds number smaller than the value of the transaction from linear to nonlinear flow (labelled as B4, B5, B6, B7 in Fig. 4). The calculated coefficients were used to predict the pressure gradients. The experimental and predicted pressure gradient versus Reynolds number is presented in Fig. 4, which shows that the predictions reproduce the experimental data.

In fact, both Ergun and Forchheimer coefficients are related to Darcy and non-Darcy terms in Forchheimer equation; they depend not only on the porous media properties but also on the fluid properties. This makes the accurate determination of the exact value, a complex and challenging process. For more reliable predictions, nonlinear regression was used only 


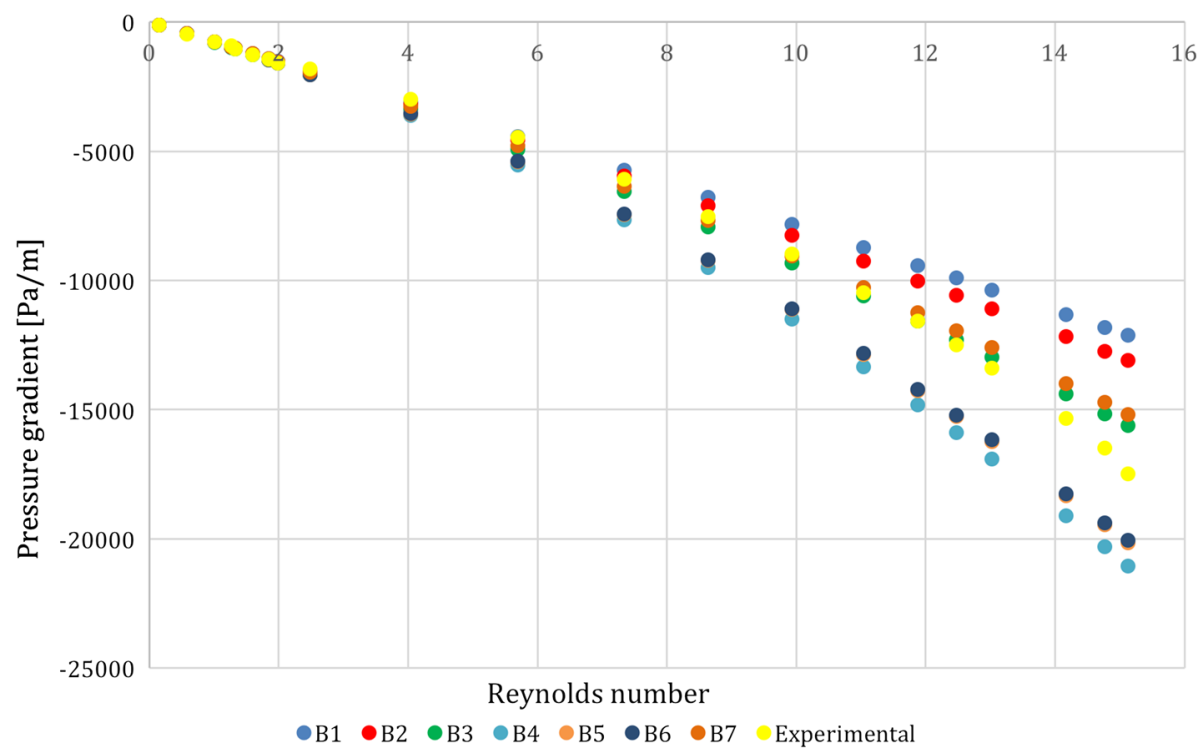

Fig. 4 Pressure gradient versus Reynolds number for different Forchheimer coefficients for $1.85 \times 10^{-3} \mathrm{~m}$ sample

on experimental data within Reynolds number (using first 9 subsets of the experiments) to calculate intrinsic permeability. We used the concept of apparent permeability. Apparent permeability is the permeability calculated for porous media in high velocities, with nonlinear flow. It can be calculated using (Barree and Conway 2004)

$$
\frac{-\Delta p}{L}=-\frac{\mu v}{k_{\mathrm{app}}}
$$

where $k_{\text {app }}$ is apparent permeability. It is defined as

$$
\frac{1}{k_{\mathrm{app}}}=\frac{1}{k}+\beta \frac{\rho v}{\mu}
$$

Apparent permeability is equivalent to permeability in Darcy's Law when the flow is within Darcy flow regime. It decreases with increasing discharge, as a result of apparent inertial effects. The intrinsic and apparent permeability values have been substituted in Eq. 4 to calculate Forchheimer coefficient for different of experiments, and it was concluded that the value of the Forchheimer coefficient varies if the nonlinearity effect is taken into account. As shown in Fig. 5, when the flow is at high velocity, Forchheimer coefficient tends to increase with Reynolds number linearly. If Forchheimer coefficient is proportional to Reynolds number, then this relation can be determined from the plots in Fig. 5, as stated in Eq. 5.

$$
\beta_{R e}=575.52 R e+159.14
$$

Forchheimer equation (i.e. Eq. 2) can be written as

$$
-\frac{\Delta p}{L}=\frac{\mu v}{k}\left(1+\beta_{R e} \frac{k \rho v}{\mu}\right)
$$

where $\beta_{R e}$ is the Forchheimer coefficient defined from Reynolds number. 


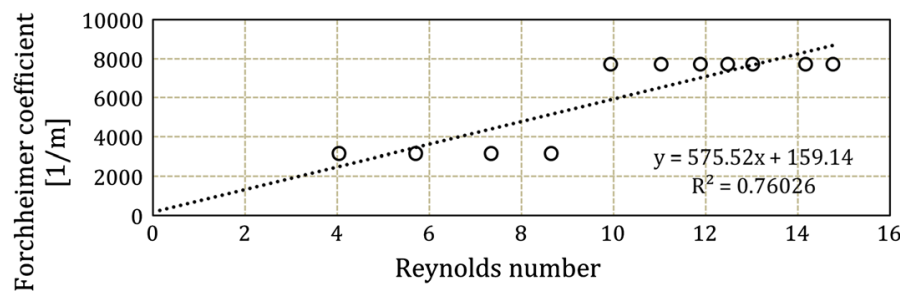

Fig. 5 Linear relationship between Forchheimer coefficient and Reynolds number for $1.85 \times 10^{-3} \mathrm{~m}$ sample

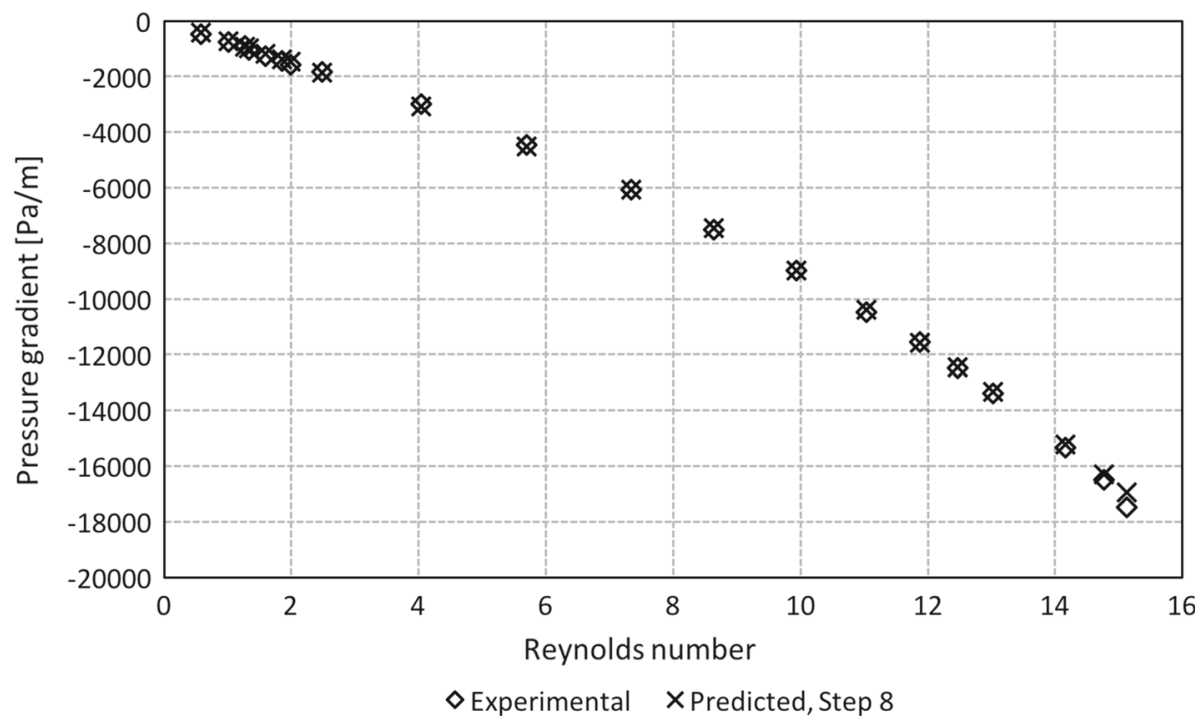

Fig. 6 Pressure gradient versus Reynolds number for $1.85 \times 10^{-3} \mathrm{~m}$ sample

The experimental data and $\beta_{R e}$ were substituted back into Eq. 6 to calculate the predicted pressure gradient; results are plotted in Fig. 6 which shows a good fit. Then, the Ergun coefficient $C_{\mathrm{E}}$ from $\beta_{R e}$ is calculated as

$$
C_{\mathrm{E} 12}=\beta_{R e} \sqrt{k}=\sqrt{k}(575.52 R e+159.14)
$$

\subsection{Dispersion}

Diffusion coefficients are calculated using a methodology presented in Chandler (2012). So we used

$$
D=\left(\frac{\sqrt{\pi}}{2 C_{\mathrm{o} . \mathrm{s}}} \frac{\mathrm{d} M_{w}}{\mathrm{~d} t^{1 / 2}}\right)^{2}
$$

where $C_{\text {o.s }}$ is the initial solute concentration within the porous medium, $\frac{\mathrm{d} M_{w}}{\mathrm{~d} t^{1 / 2}}$ is the initial slope taken from the temporal concentration profile, where $M_{w}$ is the accumulated mass of the tracer, and $t$ is the time.

In order to check the repeatability of the tracer tests, three tests for each Reynolds number were repeated. Figure 7 shows the downstream profiles for tracer tests under different 

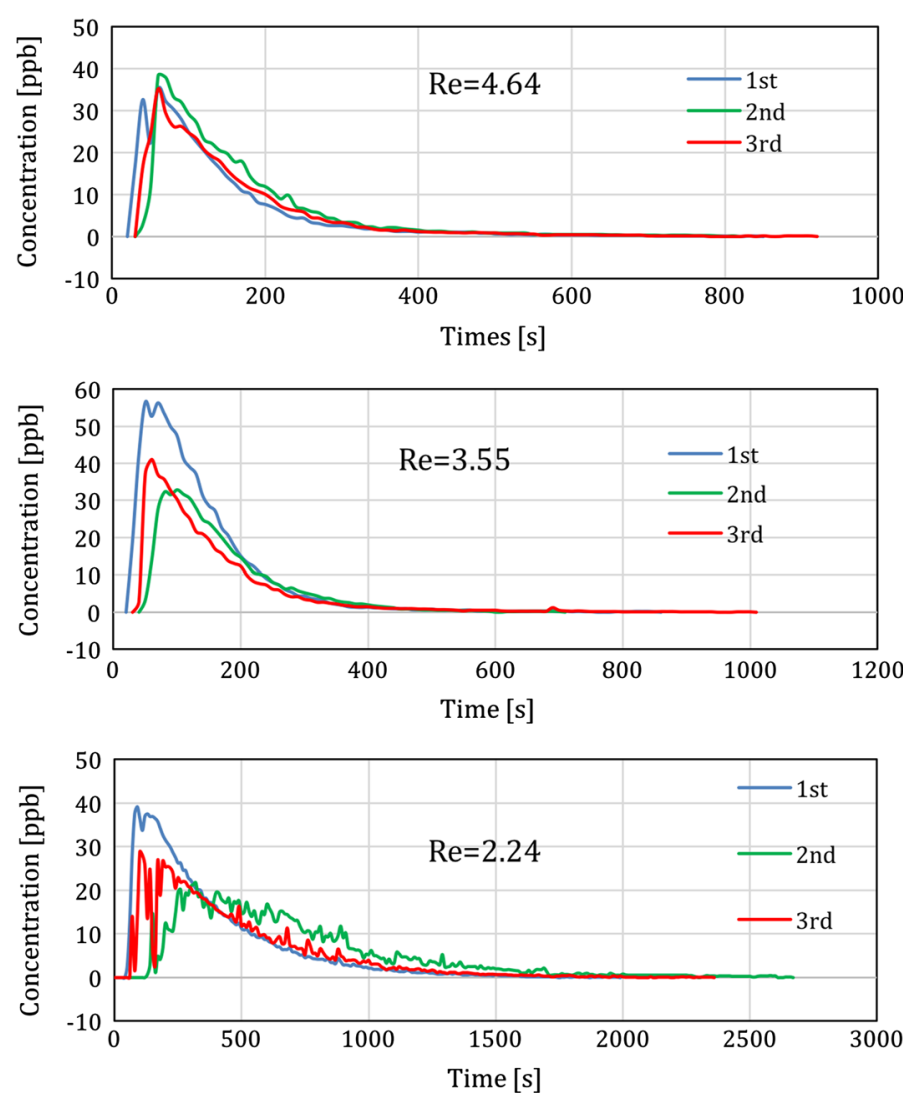

Fig. 7 Downstream profiles of the tracer tests with various Reynolds number in $1.85 \times 10^{-3} \mathrm{~m}$ sample

Reynolds numbers. It is noticeable that, for slow flow the tests are non-repeatable and different concentration profiles are achieved. This is because of the unique path for dye movement in the porous media due to unique arrangement of the spheres in each sample. As it is complicated to determine the exact pore scale structure of the porous samples, tests associated to each flow rate were repeated for three times to obtain a reliable trend representing the dispersion profile.

Figures 8 and 9 show that the spreading of the dye increases with decreasing Reynolds number. The long tailing of curves in both figures are related to the time that particles spent for macroscopic spread in low-velocity regions, which is in agreement with findings of Bijeljic and Blunt (2006). For flow within the slow flow regime, the injected dye could not be well mixed before entering the sample. Some dye stuck to the surface of the particles in porous media and could not be easily removed with the very slow flow. It needed to be continually washed off with a long duration. Therefore, tests with slower flow show longer tails.

\subsection{Effect of Flow Regime and Particle Size on Dispersion Coefficient}

As demonstrated by numerous studies, the dispersive regimes and the flow regimes in porous media can be identify, respectively, by Peclét number $\left(P e_{\mathrm{p}}\right)$ and Particle Reynolds number 


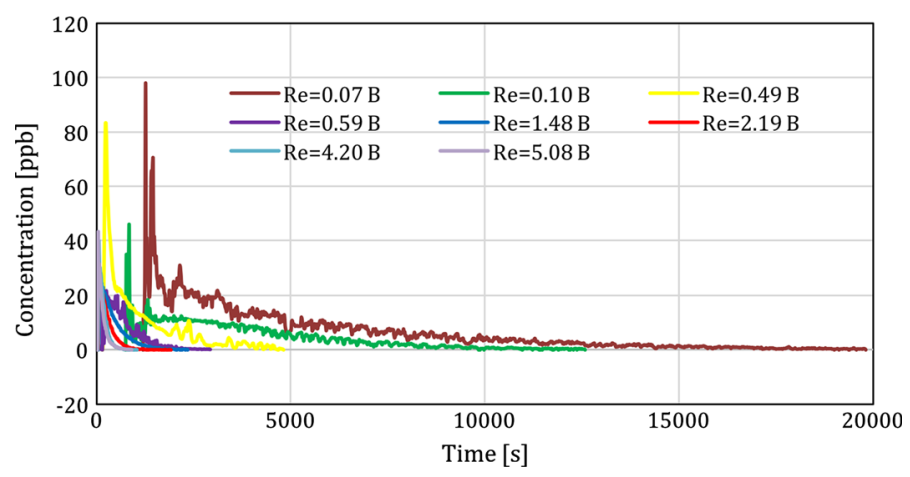

Fig. 8 Downstream (B072 Cyclops at downstream) profiles for the tracer tests under various Reynolds number in the $1.85 \times 10^{-3} \mathrm{~m}$ sample

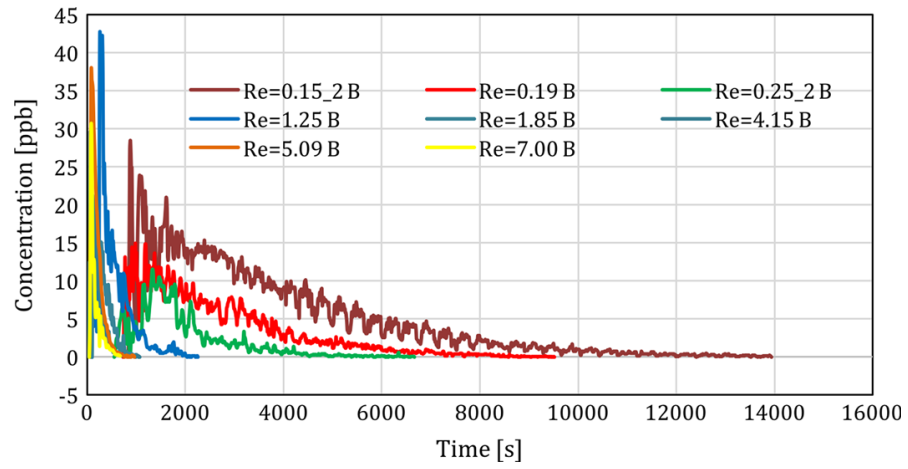

Fig. 9 Downstream (B072 Cyclops at downstream) profiles for tracer tests under various Reynolds number in the $3 \times 10^{-3} \mathrm{~m}$ sample

$\left(R e_{\mathrm{p}}\right)$. As indicated in Wood (2007), the two numbers are related through the following expressions.

$$
\begin{aligned}
R e_{\mathrm{p}}= & \frac{2}{3} \frac{\rho_{\beta}\left\langle v_{\beta}\right\rangle^{\beta} d_{\mathrm{p}}}{\mu_{\beta}}\left(\frac{\varepsilon_{\beta}}{1-\varepsilon_{\beta}}\right) \\
P e_{\mathrm{p}}= & \frac{2}{3} \frac{\left\langle v_{\beta}\right\rangle^{\beta} d_{\mathrm{p}}}{D_{A \beta}}\left(\frac{\varepsilon_{\beta}}{1-\varepsilon_{\beta}}\right) \\
& P e_{\mathrm{p}}=R e_{\mathrm{p}} S c
\end{aligned}
$$

where, $\rho_{\beta}$ represents the fluid density $\left(\mathrm{kg} / \mathrm{m}^{3}\right),\left\langle v_{\beta}\right\rangle^{\beta}$ represents the intrinsic averaged pore water velocity $(\mathrm{m} / \mathrm{s}), d_{\mathrm{p}}$ represents the particle diameter $(\mathrm{m}), \mu_{\beta}$ represents the dynamic fluid viscosity $\left(\mathrm{kg} \mathrm{m}^{-1} \mathrm{~s}^{-1}\right), \varepsilon_{\beta}$ represents the porosity, $D_{A \beta}$ represents the molecular diffusion coefficient for the solute $\left(3 \mathrm{~m}^{2} / \mathrm{s}\right)$, and $S c$ represents the Schmidt number.

The conventional dispersion regimes and the Peclét range value for water were defined as (Wood 2007):

(1) Molecular diffusion regime $\left(P e_{\mathrm{p}}<0.2\right)$ : the molecular diffusion predominates over mechanical dispersion; 


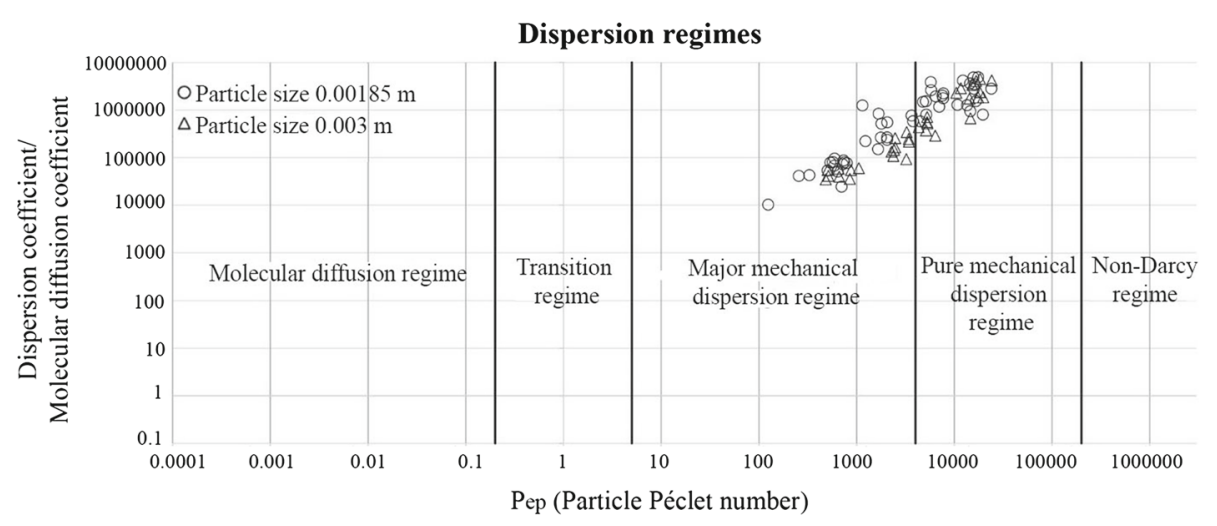

Fig. 10 Comparison of dispersion regime between particle $1.85 \times 10^{-3}$ and $3 \times 10^{-3} \mathrm{~m}$

(2) Transition regime $\left(0.2<P e_{\mathrm{p}}<5\right)$ : the molecular diffusion and mechanical dispersion have approximately the same order of magnitude;

(3) Major mechanical dispersion regime $\left(5<P e_{\mathrm{p}}<4 \times 10^{3}\right)$ : the interaction between mechanical dispersion and transverse molecular diffusion causes the spreading. The relationship between the effective dispersion coefficient $(D)$ and Peclét number is expressed by the following power low equation

$$
\frac{D}{D_{A B}}=\frac{D_{m, \mathrm{eff}}}{D_{A B}}+\alpha_{1} P e_{\mathrm{p}}^{\delta} \quad 1.1<\delta<1.2
$$

(4) Pure mechanical dispersion regime $\left(4 \times 10^{3}<P e_{\mathrm{p}}<200 \times 10^{3}\right)$ : it is characterized by power low relation of this type:

$$
\frac{D}{D_{A B}}=\alpha_{2} P e_{\mathrm{p}}^{\delta} \quad \delta=1
$$

Figure 10 shows the relation between the dispersion coefficient/molecular diffusion coefficient and Peclét number for experimental data related to the particle size of $1.85 \times 10^{-3}$ $\mathrm{m}$ and $3 \times 10^{-3} \mathrm{~m}$. It is observed that the dispersion coefficient initially increases linearly with the increases of the Reynolds number up to the condition that the value of the Reynolds number reaches to one. Considering the range Peclét number mentioned in by Wood (2007), in both cases the experimental data fall under the major mechanical dispersion regime and the pure mechanical dispersion regime. Consequently, it is not possible to use the experimental data to identify Peclét number related to the molecular diffusion regime and transition regime. For these regimes we consider the literature values. As shown in Figs. 11 and 12 the increasing trends are similar for both particle sizes. Moreover, the smaller particles have a higher velocity that increases the fluctuation in flow field and consequently the dispersion.

For the major mechanical dispersion and pure mechanical dispersion regime Eqs. (12 and 13) are determined by a linear regression between $\log \left(\frac{D}{D_{A B}}-\frac{D_{m \text {,eff }}}{D_{A B}}\right)$ and $\log \left(P e_{\mathrm{p}}\right)$ in the first case and between $\log \left(\frac{D}{D_{A B}}\right)$ and $\log \left(P e_{\mathrm{p}}\right)$ in the second one. The results are shown in Fig. 12. For the major mechanical dispersion regime, the equation in logarithmic and non-logarithmic form results:

$$
\log \left(\frac{D}{D_{A B}}-\frac{D_{\text {eff }}}{D_{A B}}\right)=1.2374 \log \left(P e_{\mathrm{p}}\right)+1.4805
$$




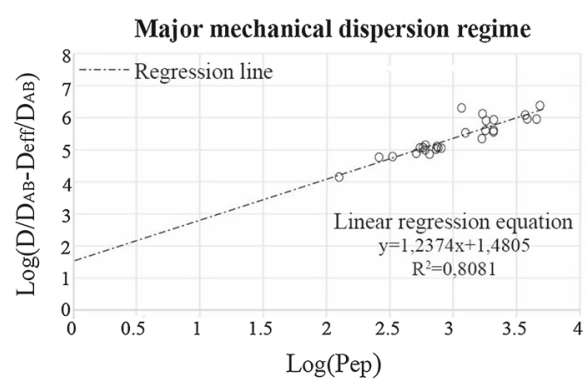

(a)

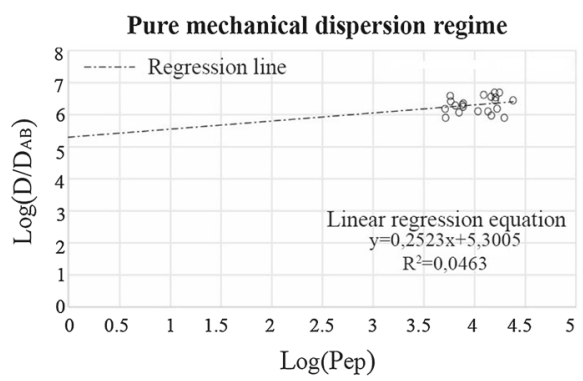

(b)

Fig. 11 Linear regression for the major mechanical dispersion regime (a) and the pure mechanical dispersion regime (b) for particle size of $1.85 \times 10^{-3} \mathrm{~m}$

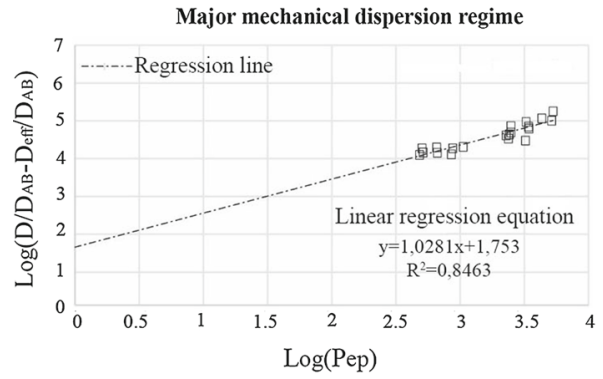

(a)

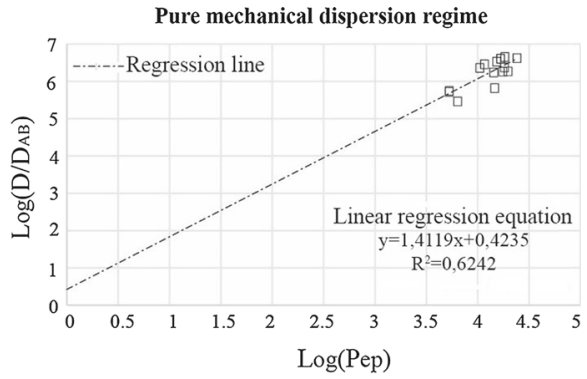

(b)

Fig. 12 Linear regression for the major mechanical dispersion regime (a) and the pure mechanical dispersion regime (b) for particle size of $3 \times 10^{-3} \mathrm{~m}$

$$
\frac{D}{D_{A B}}=\frac{D_{\mathrm{eff}}}{D_{A B}}+30.2343 P e_{\mathrm{p}}^{1.237}
$$

The $\delta$ value is 1.237 and it is slightly higher than indicated in the literature studies, where which $\delta$ is between 1.1 and 1.2.

Peclèt number corresponding to the transition from major mechanical dispersion regime to pure mechanical dispersion regime is equal to $4.78 \times 10^{3}$. It is determined considering a range of data so that the regression model provides a good estimate of the experimental data. The value matches with the one indicated by Wood (2007) under which the major mechanical dispersion regime takes place for a Peclét number between 0.2 and $5 \times 10^{3}$.

Regarding the pure mechanical dispersion regime, as shown in Fig. $12 \mathrm{~b}$ the $R^{2}$ is very low. It means that the relation (12) does not estimate correctly the experimental data in the pure mechanical regime.

For the particle size of $0.003 \mathrm{~m}$ the equation of the major mechanical dispersion regime results:

$$
\begin{gathered}
\log \left(\frac{D}{D_{A B}}-\frac{D_{\text {eff }}}{D_{A B}}\right)=1.0281 \log \left(P e_{\mathrm{p}}\right)+1.753 \\
\frac{D}{D_{A B}}=\frac{D_{\text {eff }}}{D_{A B}}+56.6239 P e_{\mathrm{p}}^{1.0281}
\end{gathered}
$$

In this case the estimated value of $\delta$ is slightly lower than the one in the literature. The transition from the major mechanical dispersion regime and the pure mechanical dispersion 


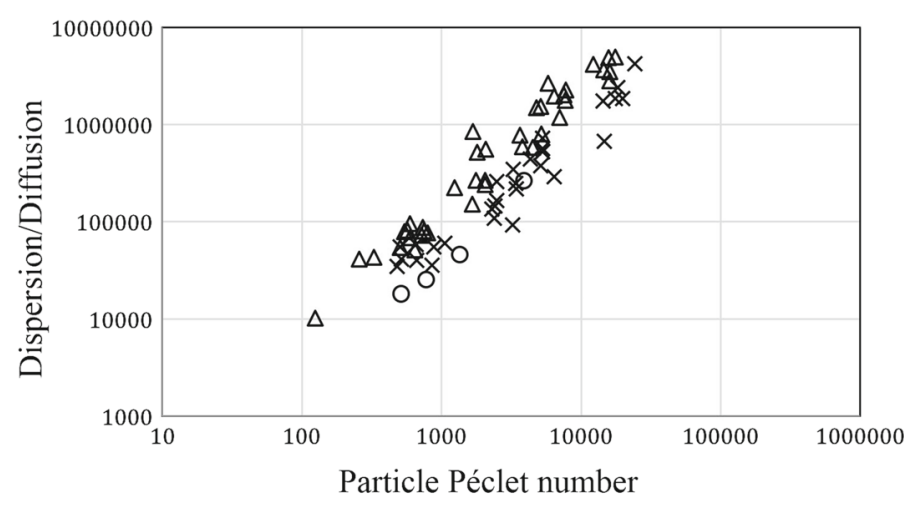

$\Delta 0.00185 \mathrm{~m} \times 0.003 \mathrm{~m} \quad 00.005 \mathrm{~m}$

Fig. 13 Dispersion coefficient/diffusion coefficient versus Péclet number for all results

regime occurs for a Peclét number of $5.28 \times 10^{3}$, a value slightly higher than the one found for the particle of $1.85 \times 10^{-3} \mathrm{~m}$. Instead, for the pure mechanical dispersion regime the relation found in the form of Eq. 13 is:

$$
\begin{gathered}
\log \left(\frac{D}{D_{A B}}\right)=1.4119 \times \log \left(P e_{\mathrm{p}}\right)+0.4235 \\
\frac{D}{D_{A B}}=2.6515 P e_{\mathrm{p}}^{1.4119}
\end{gathered}
$$

For this regime literature studies indicate a value of $\delta$ around 1. As shown before, the value found using experimental data is a little higher than 1 (Fig. 13).

As shown in Figs. 14 and 15, the increasing trends for different samples are similar. However, the sample with smaller diameter has larger dispersion/diffusion ratio and less travel time, which shows smaller particle size has more effects on shear dispersion. Longitudinal spreading is caused by the combined action of differential advection and transverse diffusion. When these two mechanisms act simultaneously, a shear-induced longitudinal dispersion process is generated. In shear flow, velocity varies in transverse direction. As the flow rate became larger and nonlinear, the difference of travel time between fine and coarse sample became smaller. The results show an agreement with Bedmar et al. (2008), which stated that due to the increase in pore-sized distribution and surface smoothness of particles the finer textured soils have higher dispersion coefficient value.

To analyse the relation between the flow and dispersion regimes, the previous results on the flow regimes obtained by Darcian Reynolds number are combined with the ones related to the dispersion regimes calculated by Peclét number. It is done for the particles of $1.85 \times 10^{-3}$ $\mathrm{m}$ and $3 \times 10^{-3} \mathrm{~m}$.

The comparison between the dispersion and flow regimes is made by referring to the Darcy velocity because it is common element between the different data set used for the analysis of the two regimes. The variables involved are related by Eqs. 9-11 and the following expression that binds Darcy velocity $(v)$ and intrinsic velocity $\left(\left\langle v_{\beta}\right\rangle^{\beta}\right)$ :

$$
\left\langle v_{\beta}\right\rangle^{\beta}=\frac{v}{\varepsilon_{\beta}}
$$

The results obtained are shown in Fig. 16 and in Tables 1 and 2. 


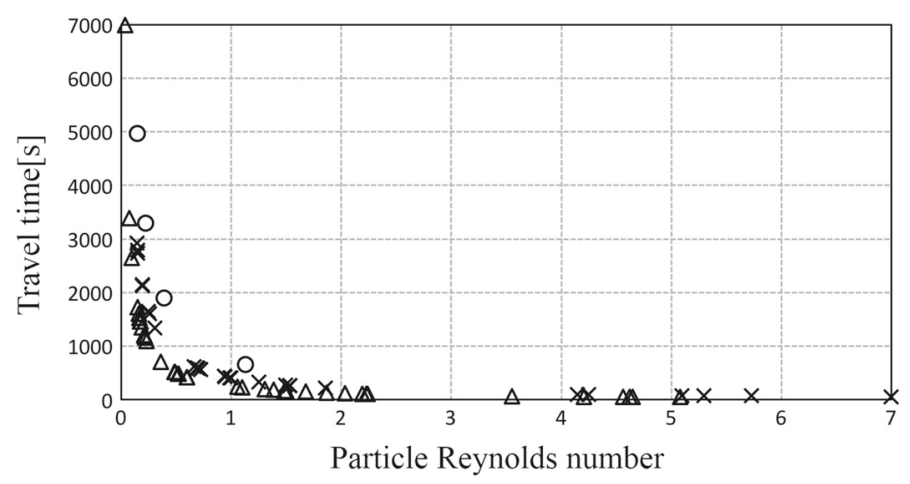

$\Delta 0.00185 \mathrm{~m} \times 0.003 \mathrm{~m} \quad 00.005 \mathrm{~m}$

Fig. 14 Travel time versus Reynolds number for all results
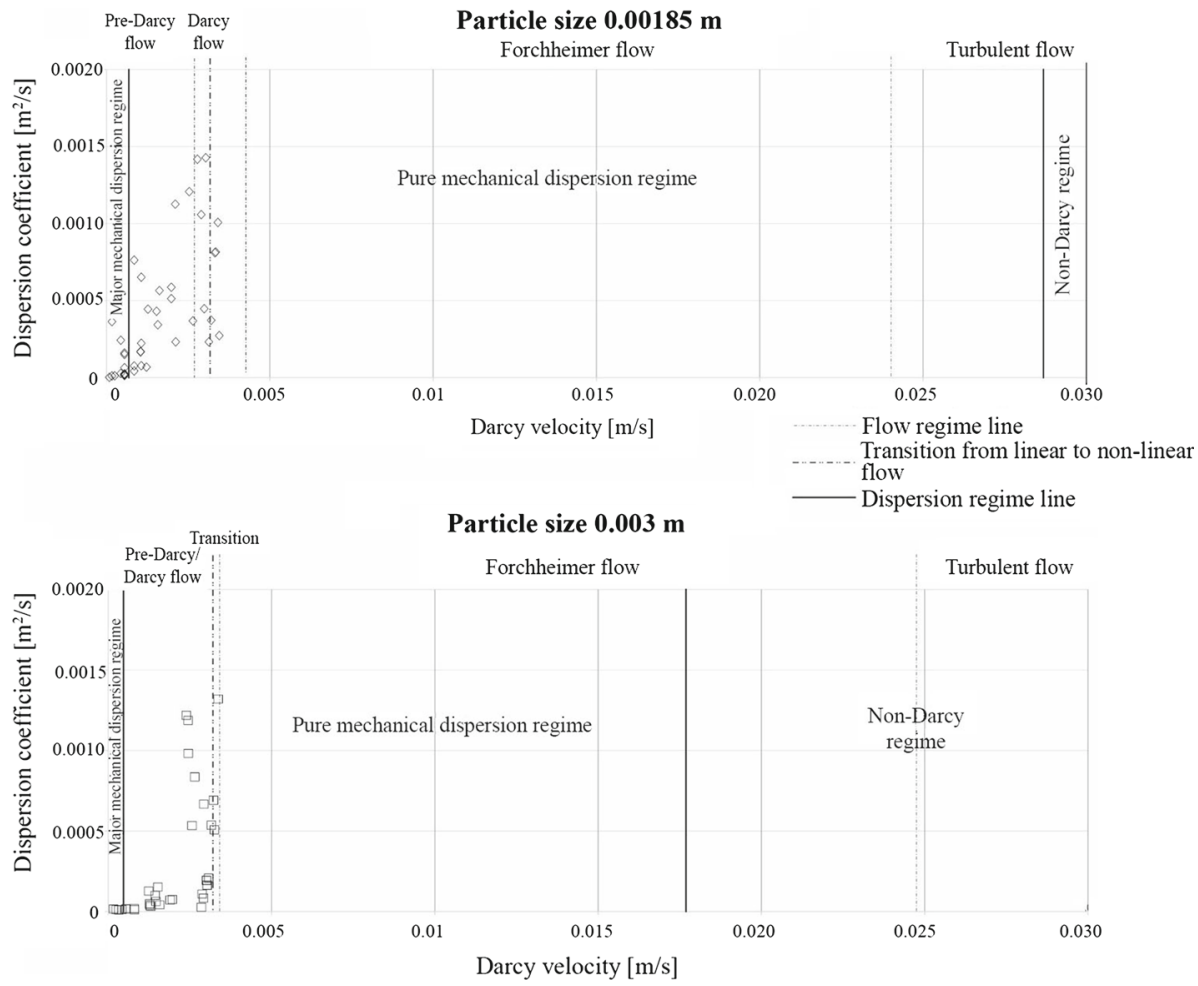

Fig. 15 Dispersion and flow regime for particle size of $1.85 \times 10^{-3} \mathrm{~m}$ and $3 \times 10^{-3} \mathrm{~m}$

As shown in Tables 1 and 2, in both cases the transition from linear to nonlinear flow occurs in the pure mechanical dispersion regime and the specific values of Darcian Reynolds number $\left(R e_{\mathrm{d}}\right)$, Peclèt number $\left(P e_{\mathrm{p}}\right)$ and Particle Reynolds number $\left(R e_{\mathrm{p}}\right)$ for the both particles size are shown below (Table 3$)$.

Ratio of dispersion to diffusion coefficient with respect to Péclet number is presented in Fig. 16 and are compared with some results from literature. It is proved that the tracer- 
Table 1 Dispersion and flow regime for particle size $1.85 \times 10^{-3} \mathrm{~m}$

\begin{tabular}{|c|c|c|c|}
\hline$P_{\text {ep }}$ & $R_{\text {ed }}$ & Dispersion regime & Flow regime \\
\hline $5<P_{\mathrm{ep}}<4.7 \times 10^{3}$ & $R_{\text {ed }}<4.16$ & $\begin{array}{l}\text { Major mechanical dispersion } \\
\text { regime }\end{array}$ & Pre-Darcy flow \\
\hline \multirow{4}{*}{$4.7 \times 10^{3}<P_{\mathrm{ep}}<2 \times 10^{5}$} & $4.16<R_{\mathrm{ed}}<6.21$ & \multirow{4}{*}{$\begin{array}{c}\text { Pure mechanical dispersion } \\
\text { regime }\end{array}$} & Darcy flow \\
\hline & $6.21<R_{\text {ed }}<7.77$ & & Transition \\
\hline & $7.77<R_{\mathrm{ed}}<44.37$ & & $\begin{array}{c}\text { Forchheimer } \\
\text { flow }\end{array}$ \\
\hline & \multirow{2}{*}{$R_{\mathrm{ed}}>44.37$} & & \multirow{2}{*}{ Turbolent flow } \\
\hline$P_{\mathrm{ep}}>2 \times 10^{5}$ & & Non-Darcy regime & \\
\hline
\end{tabular}

Table 2 Dispersion and flow regime for particle size $3 \times 10^{-3} \mathrm{~m}$

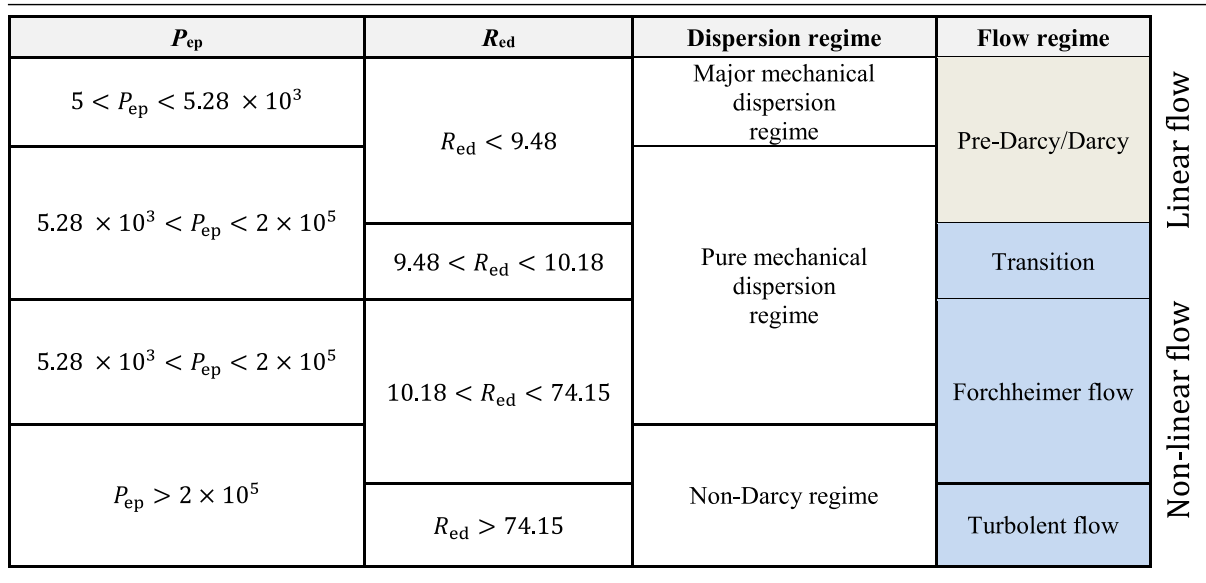

Table 3 Values of Darcian Reynolds number $\left(R e_{\mathrm{d}}\right)$, Peclèt number $\left(P e_{\mathrm{p}}\right)$ and particle Reynolds number $\left(R e_{\mathrm{p}}\right)$, corresponding to the transition from linear to nonlinear flow

\begin{tabular}{lllr}
\hline Particle size $(\mathrm{m})$ & $R e_{\mathrm{d}}$ & $P e_{\mathrm{p}}$ & $R e_{\mathrm{p}}$ \\
\hline $1.85 \times 10^{-3}$ & 6.21 & $21,871.13$ & 6.33 \\
$3 \times 10^{-3}$ & 9.48 & $35,817.37$ & 10.37 \\
\hline
\end{tabular}

determined hydrodynamic dispersion coefficient is a function of mechanical dispersion. The data show an increasing trend similar to other results from literature, but do not overlap with any other data. This is because of the dependency of dispersion coefficient to several physical properties of fluid and porous media including viscosity, density of fluid, particle size distribution and shape, fluid velocity, length of packed sample column, ratio of column diameter to particle diameter and ratio of column length to particle diameter (Delgado 2006). Any change in one of the variables would result in a different dispersion profile. For the 


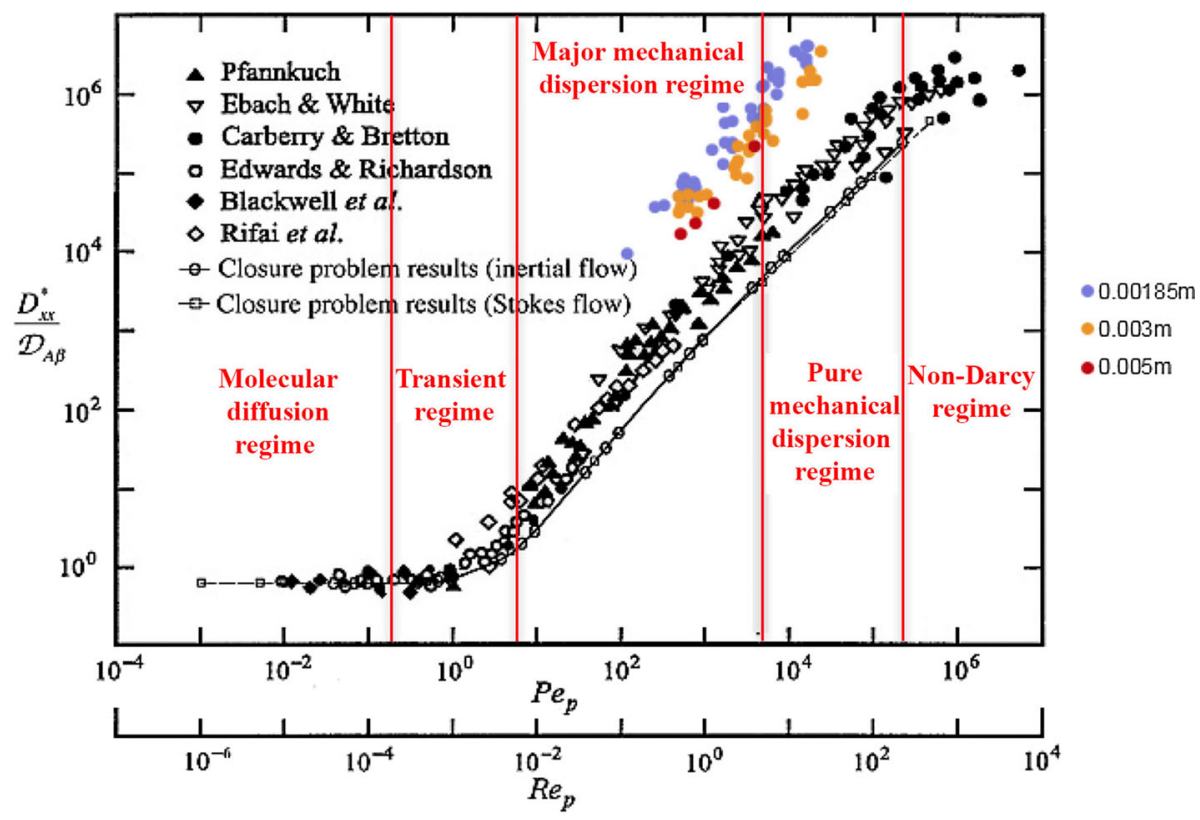

Fig. 16 Experimental results compared with dispersion regimes as a function of Péclet number

profiles presented from literature, water was considered as the fluid of interest. The diffusion coefficient of the tracer used in this research (Rhodamine WT) is $2.9 \mathrm{E}-10 \mathrm{~m}^{2} / \mathrm{s}$ as determined by Gell et al. (2001) which is different form the tracers used in the tests from the literature. Since the diffusion coefficient of Rhodamine WT is slightly larger than the other tracers, different dispersion profiles and consequently different profiles for dispersion/diffusion ratio are achieved.

\section{Conclusion}

This study focused on the impact of porous media particle size on the flow regimes and dispersion process. Water flow and dye tests through man-made cylindrical samples containing spherical glass beads were conducted to study the contribution of the flow regimes on the dispersion. Different porous media and different flow conditions were examined in this paper in order to provide a knowledge base for understanding the relationship between flow behaviour and diffusion. This understanding is essential for studying the mixing processes in porous media with particle applications in hyporheic zone modelling. Results show that by reducing particle size the magnitude of the flow velocity in that nonlinear flow occurs is reduced up to $20 \%$.

Observation within Darcian and non-Darcian regimes showed that, as the particle size increases, the diffusion effect reduces and dispersion coefficient decreases. The travel time required for dye to move through the smaller sized particles is also less than that of the larger one. This is because the finer textured sample has a more complex pore size distribution which causes a higher dispersion coefficient value. For the same Reynolds number, the dispersion coefficient of the fine particle is larger than the coarse particle. A small change in Reynolds 
number for smaller size particles results in a larger change of dispersion coefficient. This indicates that fine particles are more sensitive to flow regimes.

Acknowledgements Support to conduct this study was provided by the Monash Warwick Alliance Seed Fund.

Open Access This article is distributed under the terms of the Creative Commons Attribution 4.0 International License (http://creativecommons.org/licenses/by/4.0/), which permits unrestricted use, distribution, and reproduction in any medium, provided you give appropriate credit to the original author(s) and the source, provide a link to the Creative Commons license, and indicate if changes were made.

\section{References}

Aris, R.: On the dispersion of a solute in a fluid flowing through a tube. Proc. R. Soc. Lond. A Math. Phys. Eng. Sci. 235(1200), 67-77 (1956)

Allen, K.G., von Backström, T.W., Kröger, D.G.: Packed bed pressure drop dependence on particle shape, size distribution, packing arrangement and roughness. Powder Technol. 246, 590-600 (2013)

Barree, R., Conway, M.: Beyond beta factors: a complete model for Darcy, Forchheimer, and trans-Forchheimer flow in porous media. In: SPE Annual Technical Conference and Exhibition, Houston, Texas, USA (2004)

Bagcı, O., Dukhan, N., Özdemir, M.: Flow regimes in packed beds of spheres from pre-Darcy to turbulent. Transp. Porous Media 104, 501-520 (2014)

Bedmar, F., Costa, J.L., Giménez, D.: Column tracer studies in surface and subsurface horizons of two typic argiudolls. Soil Sci. 173(4), 237-247 (2008)

Bijeljic, B., Blunt, M.J.: Pore-scale modeling and continuous time random walk analysis of dispersion in porous media. Water Resour. Res. 42, W01202 (2006). https://doi.org/10.1029/2005wr004578

Brenner, H.: Dispersion resulting from flow through spatially periodic porous media. Philos. Trans. R. Soc. Lond. A Math. Phys. Eng. Sci. 297(1430), 81-133 (1980)

Brenner, H.: Macrotransport processes. Langmuir 6(12), 1715-1724 (1990)

Çarpinlioğlu, M.Ö., Özahi, E., Gündoğdu, M.Y.: Determination of laminar and turbulent flow ranges through vertical packed beds in terms of particle friction factors. Adv. Powder Technol. 20, 515-520 (2009)

Chandler, I.D.: Vertical Variation in Diffusion Coefficient Within Sediments. University of Warwick, Coventry (2012)

Cheng, N.S.: Wall effect on pressure drop in packed beds. Powder Technol. 210, 261-266 (2011)

Cotta, R.: Integral Transforms in Computational Heat and Fluid Flow. CRC Press, Boca Raton (1993)

Day, P.: Dispersion of a moving salt-water boundary advancing through saturated sand. EOS Trans. AGU 37(5), 595-601 (1956)

De Josselin de Jong, G.: Longitudinal and transverse diffusion in granular deposits. Eos Trans. AGU 39(1), 67-74 (1958)

Delgado, J.: A critical review of dispersion in packed beds. Heat Mass Transf. 42(4), 279-310 (2006)

Dentz, M., Cortis, A., Scher, H., Berkowitz, B.: Time behavior of solute transport in heterogeneous media: transition from anomalous to normal transport. Adv. Water Resour. 27, 155-173 (2004)

Erdim, E., Akgiray, Ö., Demir, İ.: A revisit of pressure drop-flow rate correlations for packed beds of spheres. Powder Technol. 283, 488-504 (2015)

Evans, E., Kenney, C.: Gaseous dispersion in packed beds at low Reynolds numbers. Trans. Inst. Chem. Eng. 44, T189-T197 (1966)

Fand, R.M., Kim, B.Y.K., Lam, A.C.C., Phan, R.T.: Resistance to the flow of fluids through simple and complex porous media whose matrices are composed of randomly packed spheres. J. Fluids Eng. 109(3), 268-273 (1987)

Forchheimer, P.: Wasserbewegung durch boden. Z. Ver. Deutsch. Ing. 45(1782), 1788 (1901)

Gell, C., Brockwell, D.J., Beddard, G.S., Radford, S.E., Kalverda, A.P., Smith, D.A.: Accurate use of single molecule fluorescence correlation spectroscopy to determine molecular diffusion times. Single Mol. 2(3), 177-181 (2001)

Hamielec, A., Hoffman, T., Ross, L.: Numerical solution of the Navier-Stokes equation for flow past spheres: part I. Viscous flow around spheres with and without radial mass efflux. AIChE J. 13(2), 212-219 (1967a)

Hamielec, A., Johnson, A., Houghton, W.: Numerical solution of the Navier-Stokes equation for flow past spheres: part II. Viscous flow around circulating spheres of low viscosity. AIChE J. 13(2), 220-224 (1967b)

Han, N., Bhakta, J., Carbonell, R.: Longitudinal and lateral dispersion in packed beds: effect of column length and particle size distribution. AIChE J. 31(2), 277-288 (1985) 
Haserta, M., Bernsdorfa, J., Rollera, S.: Lattice Boltzmann simulation of non-Darcy flow in porous media. Procedia Comput. Sci. 4, 1048-1057 (2011)

Hofmann, S., Bufe, A., Brenner, G., Turek, T.: Pressure drop study on packings of differently shaped particles in milli-structured channels. Chem. Eng. Sci. 155, 376-385 (2016)

Kececioglu, I., Jiang, Y.: Flow through porous media of packed spheres saturated with water. J. Fluid Eng. 116, 164-170 (1994)

LeClair, B., Hamielec, A.: Viscous flow through particle assemblages at intermediate Reynolds numbers. Steady-state solutions for flow through assemblages of spheres. Ind. Eng. Chem. Fund. 7(4), 542-549 (1968)

Liang, S.: Investigation of Nonlinear Flow and Dispersion in Porous Media. MSc Thesis, University of Warwick, UK (2017)

Majdalani, S., Chazarin, J.P., Delenne, C., Guinot, V.: Solute transport in periodically heterogeneous porous media: importance of observation scale and experimental sampling. J. Hydrol. 520, 52-60 (2015)

Mickley, H., Smith, K., Korchak, E.: Fluid flow in packed beds. Chem. Eng. Sci. 20(3), 237-246 (1965)

Montroll, E., Weiss, G.: Random walks on lattices. II. J. Math. Phys. 6(2), 167-181 (1965)

Nezhad, M.M.: Stochastic Finite Element Modeling of Flow and Solute Transport in Dual Domain System. $\mathrm{PhD}$ thesis, University of Exeter, UK (2010)

Nezhad, M.M., Javadi, A.A.: Stochastic finite-element approach to quantify and reduce uncertainty in pollutant transport modeling. J. Hazard. Toxic Radioact. Waste 15(3), 208-215 (2011)

Nezhad, M.M., Javadi, A.A., Rezania, M.: Finite element modelling of contaminant transport considering effects of micro and macro heterogeneity of soil. J. Hydrol. 404(3-4), 332-338 (2011)

Nezhad, M.M., Javadi, A.A., Al-Tabbaa, A., Abbasi, F.: Numerical study of soil heterogeneity effects on contaminant transport in unsaturated soil; model development and validation. Int. J. Numer. Anal. Met. 37(3), 278-298 (2013)

Norouzi, A.M., Siavashi, M., Soheili, A.R., Khaliji Oskouei, M.: Experimental investigation of effects of grain size, inlet pressure and flow rate of air and argon on pressure drop through a packed bed of granular activated carbon. ICHMT 96, 20-26 (2018)

Petrasch, J., Meier, F., Friess, H., Steinfeld, A.: Tomography based determination of permeability, DupuitForchheimer coefficient, and interfacial heat transfer coefficient in reticulate porous ceramics. Int. J. Heat Fluid Flow 29, 315-326 (2008)

Prausnitz, J., Wilhelm, R.: Turbulent concentration fluctuations in a packed bed. Ind. Eng. Chem. 49(6), 978-984 (1957)

Reddy, S., Schmid, P., Baggett, J., Henningson, D.: On the stability of streamwise streaks and transition thresholds in plane channel flows. J. Fluid Mech. 365, 269-303 (1998)

Sanchez-Palencia, E.: Vibration of mixtures of solids and fluids. In: Non-Homogeneous Media and Vibration Theory. Lecture Notes in Physics, vol. 127, pp. 158-190. Springer, Berlin (1980)

Scheidegger, A.: Statistical hydrodynamics in porous meida. J. Appl. Phys. 25(8), 994-1001 (1954)

Scheidegger, A.: General theory of dispersion in porous media. J. Geophys. Res. 66(10), 3273-3278 (1961)

Shapiro, M., Brenner, H.: Taylor dispersion of chemically reactive species: irreversible first-order reactions in bulk and on boundaries. Chem. Eng. Sci. 41(6), 1417-1433 (1986)

Shapiro, M., Brenner, H.: Dispersion of a chemically reactive solute in a spatially periodic model of a porous medium. Chem. Eng. Sci. 43(3), 551-557 (1988)

Simmons, C.S.: A stochastic-convective transport representation of dispersion in one-dimensional porous media systems. Water Resour. Res. 18(4), 1193-1214 (1982)

Slichter, C.: Field Measurement of the Rate of Movement of Underground Water. Water Supply Paper. USGS Publications Warehouse, Govt. Print. Off., https://doi.org/10.3133/wsp140 (1905)

Taylor, G.: Dispersion of soluble matter in solvent flowing slowly through a tube. Proc. R. Soc. Lond. A Math. Phys. Eng. Sci. 219(1137), 186-203 (1953)

van der Merwe, D., Gauvin, W.: Velocity and turbulence measurements of air flow through a packed bed. AIChE J. 17(3), 519-528 (1971)

Vollmari, K., Oschmann, T., Wirtz, S., Kruggel-Emden, H.: Pressure drop investigations in packings of arbitrary shaped particles. Powder Technol. 271, 109-124 (2015)

Whitaker, S.: The Forchheimer equation: a theoretical development. Transp. Porous Media 25(1), 27-61 (1996)

Wood, B.D.: Inertial effects in dispersion in porous media. Water Resour. Res. 43, 12-16 (2007)

Zeng, Z., Grigg, R.: A criterion for non-Darcy flow in porous media. Transp. Porous Media 63, 57-69 (2006) 